\title{
Bounded Rational Onlinè Bin Packing Solution Using Garbage Can Model ${ }^{\dagger}$
}

\author{
Qiang WeI*, Tetsuo SaWARagi* and Yajie Tian*
}

\begin{abstract}
Bin Packing(BP) problem is one kind of typical combinatorial optimization problems concerned about packing a certain amount of different items into the minimal number of bins under specific constraints. In this paper, an organizational model called Garbage Can Model(GCM) is adopted to deal with a relaxed online BP problems. In GCM, the organizational structure and fluidities of problems and resources provide many optimization and re-combination opportunities for arriving items in stream. Different from traditional online BP algorithms, which usually result in a less-optimal solution in pursuing an extremely online executing speed, GCM-based algorithm achieves finding a bounded optimal solution for a relaxed online BP in a reactive fashion through seeking for a solution as optimal as possible as far as the time is allowed.
\end{abstract}

Key Words: Garbage Can Model(GCM), bin packing(BP), bounded rationality, optimization

\section{Introduction and Definitions}

Bin Packing(BP) problem is a kind of typical combinatorial optimization problems, which consists of packing a certain amount of different items into the minimal number of bins under specific constraints. More formally,

DEFINITION Given a finite set $L=\left\{a_{1}, a_{2}, \ldots, a_{n}\right\}$ of "items" and a rational "size", size $\left(a_{j}\right) \in(0,1]$, for each item $a_{j} \in L$, find a partition of $L$ into disjoint subsets $L_{1}, L_{2}, \ldots, L_{k}$ such that the sum of the sizes of the items in each $L_{i}$ is no greater than 1 and such that $k$ is as small as possible. ${ }^{2)}$

An online bin packing problem requires the packing of the $i$ th item without information on the sizes or the number of the subsequently incoming input items. Until now, most online algorithms(e.g., HARMONIC ${ }^{5)}$ ) do not permit moving items from the bins once they are assigned to them. Although such constraint is from the requirement of fast online executing speed, it sometimes leads to a less-optimal solution.

In this paper, we follow Gambost's ideas ${ }^{1)}$ and interpret the online property of $\mathrm{BP}$ in a more relaxed way, in which items' moving among the assigned bins(i.e., re-allocation) are possible. Such a strategy is suitable for the online problems in which the bins generated out of the system are not executed immediately, but there exists some time allowance, or time buffer, between the time the bins are generated and the time it is finalized for its execution. This time gap enables us to deliberate and seek for more optimal solutions by using an optimization algorithm.

There are many real-life situations where the rearrangement of an allocated item is allowed and this affects the

\footnotetext{
$\dagger$ Presented in SCI 2003, Sendai $(2003 \cdot 11)$

* Department of Precision Engineering, Graduate School of Engineering, Kyoto University, Sakyo-ku, Kyoto

(Received January 21, 2004)
}

cost of the resolution process. For example, several parallel machines are used to pack some online arriving items into some fixed size bins. Every item has a different size. An item will wait a short time(i.e., the time gap) for packaging after it has been allocated on a machine's band carrier. This time gap changes with the variation of the band carrier's length and velocity(i.e., how many items should be processed in an hour or in a minute). In a time gap, items can be moved from a band carrier to another. The objective is to pack these items into boxes efficiently. In order to solve this kind of problems, we introduce an organizational model - Garbage Can Model(GCM).

\section{Garbage Can Model}

$\mathrm{GCM}^{4)}$ was originally introduced in a field of organization theory for explaining organized anarchies. According to Cohen's model, there are four components

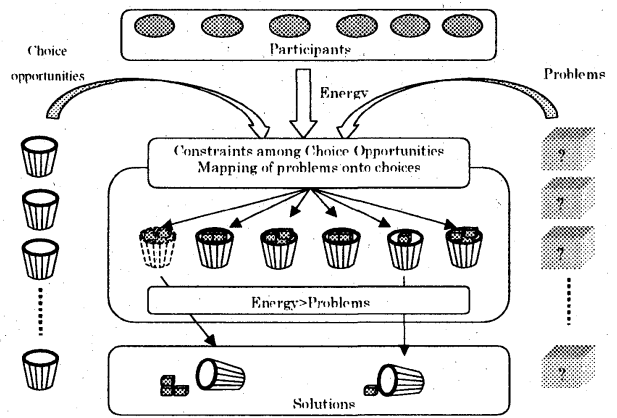

Fig. 1 Organizational mode in Garbage Can Model in GCM, as shown in Fig. 1:

Problems: The problems to be solved.

Choice opportunities: Occasions for solving problems.

Energies from participants: The resources are used to solve problems.

Solutions: Decisions output from garbage cans.

In GCM, a choice opportunity is viewed as a garbage can into which various kinds of problems and energies are dumped by participants. Participants also provide ener- 
gies for solving the problems. Problems and choice opportunities are time-serial streams and flow into the system. When some criteria are satisfied, a decision is made and this garbage can is removed from the environment.

The most important character of GCM is that problems and energies are allowed to move to another garbage can anytime whenever some criteria are satisfied(i.e., problems may disappear from a choice opportunity and participants may come and go ${ }^{3)}$. In this way, problems in each garbage can are solved not only by a participant's own efforts, but also by the change of problem solving status in other garbage cans as well as by the external environment from which the problems are thrown into the cans. In another words, problem solving in GCM may not only depend on the problem solver's own optimizing efforts, but also depend on the problem solving activity and/or status performed so far. Incomplete or partial solutions that are derived but not appropriate as final solutions can be efficiently utilized without make them invalid.

\section{Simulation and Analysis}

When we use GCM to solve a BP problem, problems, choice opportunities, energies and solutions in GCM are mapped as items, empty bins, space assigned in bins and full bins, respectively.

In a relaxed online BP, empty bins and items (whose value is less than 1) flow into the system in time series. The sum of items in each bin should not exceed 1 . The energy is considered as the space of carrying capacity in each space bin, which is consumed by the items allocated to this bin. Items can be shifted from a bin to another. When the loading ratio of the items to the bin is greater than a given value $R$, this bin is viewed as a full bins and will be dumped out of the system. At this time, the position it occupied will be used as another empty bin.

All bins are divided into three states: inactive bins, active bins and dumped bins. Inactive bins are the bins that stay in the queue and wait to be thrown into the system; active bins are the bins that have been thrown into the system. Dumped bins are the bins that are full of items and have been dumped, and the items wherein cannot be moved to other bins any more.

When a new item flows into system, the following steps will be carried out sequencely:

Step 1 Allocated by oversight. If the item can be allocated into a bin, and this bin becomes full resulting in being dumped out of the system after this allocation, then allocate the item into this bin without considering other (better) solutions; otherwise go to Step 2.
Step 2 Allocated by flight. If this item can be allocated to a bin (e.g., bin.1) through shifting an item (e.g., itemA) of bin1 to another bin (e.g., bin2), and this kind of shift results in a bin(either bin1 or bin2) that becomes full and is going to be dumped out of the system, then shift item A from bin1 to bin2(shown in Fig. 2) and allocate the arriving item into bin1; otherwise go to Step 3;

Step 3 Allocated by resolution. If the arriving item can be allocated into bins, allocate it to the bin that has the maximum height; otherwise go to Step 4;

Step 4 Create a new bin and allocate the item in it.

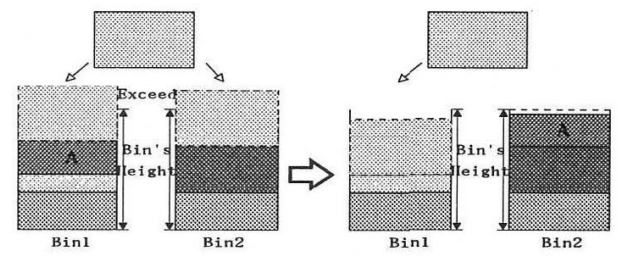

Fig. 2 Shifting item A from bin1 to bin2 if an decisions can be made on bin2'

Oversight is a fast decision-making strategy between deliberation/optimization and solution costs. An arriving item will be allocated into a bin as soon as possible if this bin is full after allocating this item. We do not try to deliberately search which bin has the maximum loading ratio after allocating this item. This aspect seems to be irrational, but a human expert is actually adopting such a strategy. This strategy can be regarded as boundedrational if we consider the tradeoffs between the easiness of getting satisfactory and the cost needed by deliberately optimization. In our problem, such strategy rapidly decreases the number of active bins co-existence in the system and keeps the system in a compact optimizing scope. It decreases the computational complexities consumed in searching the most optimal bin at the cost of a little loading quality. Oversight does not affect the total loading ratio too much, because although the bin we found may be not the most optimal one, it is an nearly optimal one which has a loading ratio more than $R$ (e.g., $R=0.9$ ).

Flight provides many re-allocation and optimization opportunities for finding a better solution. If an arriving item cannot be allocated by oversight, it will be allocated through reallocating one of the items in an active bin to another active bin.

In such flight, an optimal solution is not only contributed by the status of an individual bin, but also by the status of all the co-existing active bins. That is, although the item can only be allocated into a bin(e.g., $\operatorname{bin} 1)$, other bins'(e.g., bin.2) status/situations do affect whether bin1 can hold the item or not.

The time gap affects the optimization quality consider- 
ably. As we mentioned in the first section, the time gap enables finding an optimization results through flight. If such a time gap is not allowed at all, a real-time and/or online bin-packing algorithm is needed such as HARMONIC, but it only generates less-optimal solutions. On the other hand, there might be possible to intermittently adopt off-line optimization such as a tabu search algorithm. In that case, however, the coming items thrown into during the off-line optimization cannot used in the ongoing problem solving and they have to wait for being allocated into bins until the next off-line optimization iteration is resumed. Moreover, the solution obtained in the previous off-line optimization iteration has nothing to do with the one obtained in the subsequent iteration. Our proposing method can overcome such a discontinuity of problem solving and can obtain the bounded optimal solution catching up with currently incoming items and opportunistically utilizing the existing repertoire of solutions that are waiting for being executed. This is unique properties of our GCM method and is a major difference from the off-line optimization technique.

Sometimes, the time gap is not enough for flight. In flight, the re-allocation searching process will not stop until a solution is found. In the worst case(i.e.., there is no available item-bin pair for flight), this searching process consumes much time because it must traverse(i.e., browse) all the items existing in active bins. If the time consumed in a flight searching is greater than the given time gap, this flight will be interrupted. In this case, the item will be randomly allocated into an available bin without considering loading quality. Therefore, the solution of GCM also depends on the time gap given by users.

\section{Simulation results}

We compared GCM with one of famous online BP algorithms called HARMONIC ${ }^{5)}$ through simulating the allocation of 1000 randomly generated items into bins. The hardware environment is Dell INSPIRON 4000, CPU speed is $1 \mathrm{G} \mathrm{Hz}$. We set the active bins to $10(M=10)$ in HARMONIC and attained an average loading ratio of 0.7640 . In GCM, we set $R=0.9$ and the maximum count of active bins is also 10 . If the number of active bins exceeds 10 , the bin with the maximum loading height will be dumped, no matter does its height is greater than $R$ or not. As we mentioned, the solutions of GCM(i.e., average loading ratio, $\left.R_{a}\right)$ depends on the available time $\operatorname{gap}\left(T_{g}\right.$, measured by CPU clock units). We show this variation of $R_{a}$ with $T_{g}$ in Table 1.

Comparing the results with HARMONIC, we can see
Table 1 The average loading ratio changes with time gap

\begin{tabular}{|c|c|c|c|c|c|c|}
\hline$T_{g}$ & 1 & 3 & 5 & 7 & 10 & 20 \\
\hline$R_{a}$ & 0.5438 & 0.7460 & 0.8219 & 0.8723 & 0.9028 & 0.9212 \\
\hline
\end{tabular}

that when $T_{g}$ are no less than $5 \mathrm{CPU}$ time(about 0.001 sec.), GCM obtains a more optimal solution than traditional HARMONIC algorithm. Such result shows that GCM needs only an ultrashort time gap for searching an optimal solution in our simulation. This is due to the fact that the computation complexity consumed on the flight among active bins is partially decreased by the oversight in GCM. Fig. 3 shows the loading ratio of bins which are generated by $\operatorname{GCM}\left(T_{g}=20\right)$ and by HARMONIC. The horizontal axis is the No. of generated bins and the vertical axis is the loading ratio of each bin. In comparing the loading ratio of these bins, we can see that most of bins in GCM have a better solution than those in HARMONIC when the time gap is enough for flight.

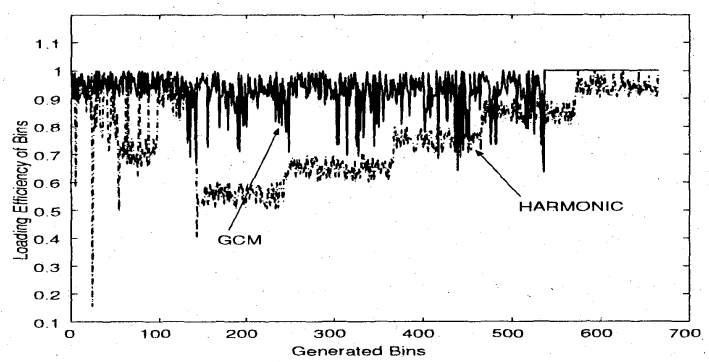

Fig. 3 Comparison of loading ratio of the bins generated by $\operatorname{GCM}\left(T_{g}=20\right)$ and by HARMONIC

\section{Conclusion}

This paper introduced an organizational methodology - GCM into a relaxed online BP problem. Our analysis and results show GCM is able to find a "more" optimal solution than traditional online algorithm for a bounded rational $\mathrm{BP}$ problem. In GCM, the arriving items are solved not only by the optimization on an individual bin, but also by the change of the status in other bin.

\section{References}

1) Giorgio Gambost, Albertto Postiglione and Maurizio Talamo: Algorithms for the relaxed online bin packing; Society for Industrial and Applied Mathematics, 1532/1551 (2000)

2) M. R. Garey and D.S. Johnson: Computers and Intractability: A Guide to the Theory of NP-completeness, Freeman, San Francisco, CA (1979)

3) Tetsuo Sawaragi and Kiyoaki Murasawa: Socio-Technical Model of Complexity, Ambiguity and Emergence in Team Situation Awareness; Proc. of the Society for Computer Simulation International 2000 Summer Computer Simulation Conference, July 16-20 (2000)

4) M.D. Cohen, J.G. March and J.P. Olsen: A Garbage Can Model of Organizational Choice; Administrative Science Quarterly, 17-1 (1972)

5) C.C. Lee and D.T. Lee: A simple on-line bin-packing algorithm; Journal of the ACM, 32, Issue 3, 556/572 